\title{
Percutaneous approach to a tight post-isthmic aortic coarctation: a case report and literature review.
}

\author{
Francesca Cortese ${ }^{1 *}$, Michele Gesualdo ${ }^{1}$, Tommaso Acquaviva ${ }^{2}$, Annamaria Cortese ${ }^{3}$, \\ Emanuela De Cillis², Marco M.Ciccone ${ }^{1}$, Alessandro Santo Bortone ${ }^{2}$
}

\begin{abstract}
${ }^{1}$ Cardiovascular Diseases Section, Department of Emergency and Organ Transplantation (DETO), University of Bari, Bari, Italy.
${ }^{2}$ Cardiac Surgery Diseases Section, Department of Emergency and Organ Transplantation (DETO), University of Bari, Bari, Italy.

${ }^{3}$ Cerebrovascular Diseases and Neurorehabilitation Department, San Camillo Hospital, Venezia Lido, Italy.
\end{abstract}

\section{DOI Name}

http://dx.doi.org/10.3126/jaim.v5i2.17353

\section{Keywords \\ aortic coarctation; percutaneous correction, \\ covered stent \\ Citation \\ Francesca Cortese, Michele Gesualdo, Tommaso \\ Acquaviva, Annamaria Cortese, Emanuela De Cillis, Marco M Ciccone, Alessandro Santo Bortone. Percutaneoud approach to a tight post-isthmic aortic coarctation: a case report and literature reiview. Journal of Advances in Internal Medicine 2016;05(02):41-45}

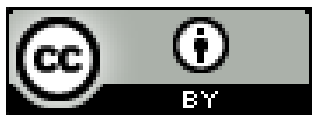

This work is licensed under a Creative Commons Attribution 3.0 Unported License.

\begin{abstract}
A 17 years-old boy with hypertension underwent cardiology assessment for episodes of dyspnoea and palpitations. Cardiac angiography showed post-istmic severe aortic coarctation. The malformation was successful treated by implanting a covered stent in aorta. The manuscript describes in detail this case and analyzes the available literature on the topic.
\end{abstract}

\section{INTRODUCTION}

Coarctation of aorta ( $\mathrm{COA})$ is a relatively common defect that accounts for $5-7 \%$ of all congenital heart defects, its incidence being of 3 new-borns every 1000 birth [1].

CoA clinical presentation varies according to its anatomic features. Young patients may present in the first few weeks of life with acute cardiac failure, acidosis, shock, and deterioration coincides with closure of the patent ductus arteriosus (Botallo's ductus). Less severe manifestations can remain asymptomatic and patients often present in childhood or early adolescence with hypertension or a murmur. Treatment can be variable according to the age of onset and severity of the coarctation. Surgical relief of the aortic obstruction and catheter interventional techniques (balloon angioplasty and stents) are available alternatives. Surgical repair is chosen when there is a complex anatomy or associated cardiac defects, it is also preferred in new-borns or infants since balloon angioplasty is associated with some risks like long term development of aneurisms, re-coarctation with the need of re-stenting and limitations related to the small calibre of the children vessels. Percutaneous procedures represent, instead, the better approach in older children, adolescents and young adults with simple native and juxta-ductale coarctation representing a less invasive alternative to surgery with good long-term results [2]. In this manuscript, we are going to illustrate a case report of a percutaneous repair of a tight post isthmic native aortic

\footnotetext{
* Corresponding author Francesca Cortese, MD

Cardiovascular disease Section Department of Organ and Trasplantation University of Bari, Italy email:francescacortese@hotmail.it
} 
coarctation in a teenager. We also reviewed the available literature on this topic.

\section{CASE REPORT}

A 17 years old boy was referred to our cardiology unit after an incidental finding of post-isthmic aortic coarctation, confirmed with echocardiography. He was an agonist football player which was addressed to a cardiology clinic for palpitations and exercise associated dyspnoea. In his past medical history, he suffered from hypertension and he didn't have other relevant medical conditions. On admission to our unit his whole clinical examination, blood results and chest XR were normal, ECG showed sinus rhythm with long PR interval, signs of left ventricular hypertrophy and ventricular repolarization abnormalities (figure 1).

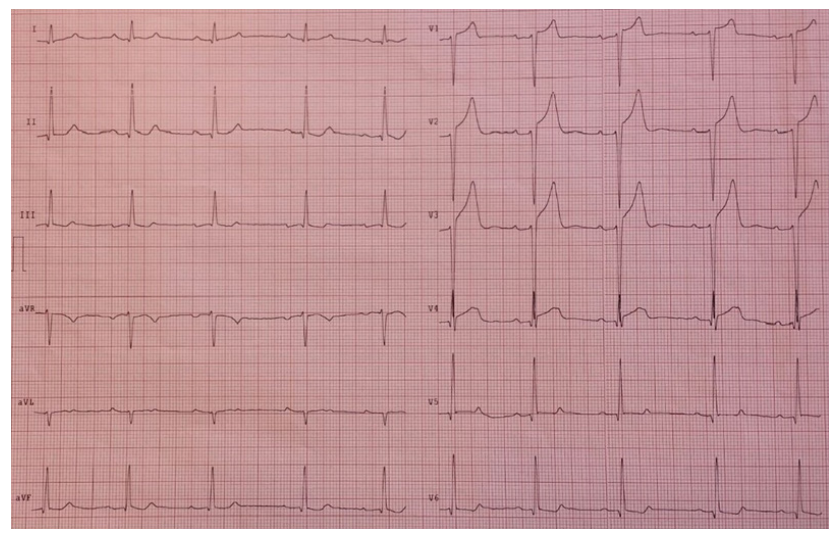

Figure 1: Electrocardiographic abnormalities showing left ventricular hypertrophy (see text).

Eventually the echocardiography revealed severe native post-isthmic aortic coarctation with a maximum gradient of $65 \mathrm{mmHg}$. The left ventricle showed a moderate degree of uniform hypertrophy, with concentric remodelling and 1st degree diastolic dysfunction, identified the peak early filling velocity $(E) /$ late diastolic filling velocity $(A)$ ratio less than 1 assessed by pulsed-wave Doppler in the apical 4-chamber view to obtain mitral and tricuspid inflow velocities [3], normal cavitarian dimension and preserved systolic function. The aortic transvalvular gradient was of about $45 \mathrm{mmHg}$, with the maximum diameter of ascending aorta of $20 \mathrm{~mm}$ and that of aortic arch of $15 \mathrm{~mm}$.

Therefore, this young patient underwent a cardiac angiography which showed a tight post-isthmic aortic coarctation, with a virtual lumen and collateral circuits connecting the two tracts of the aorta before and after the coarcted segment (figure 2).

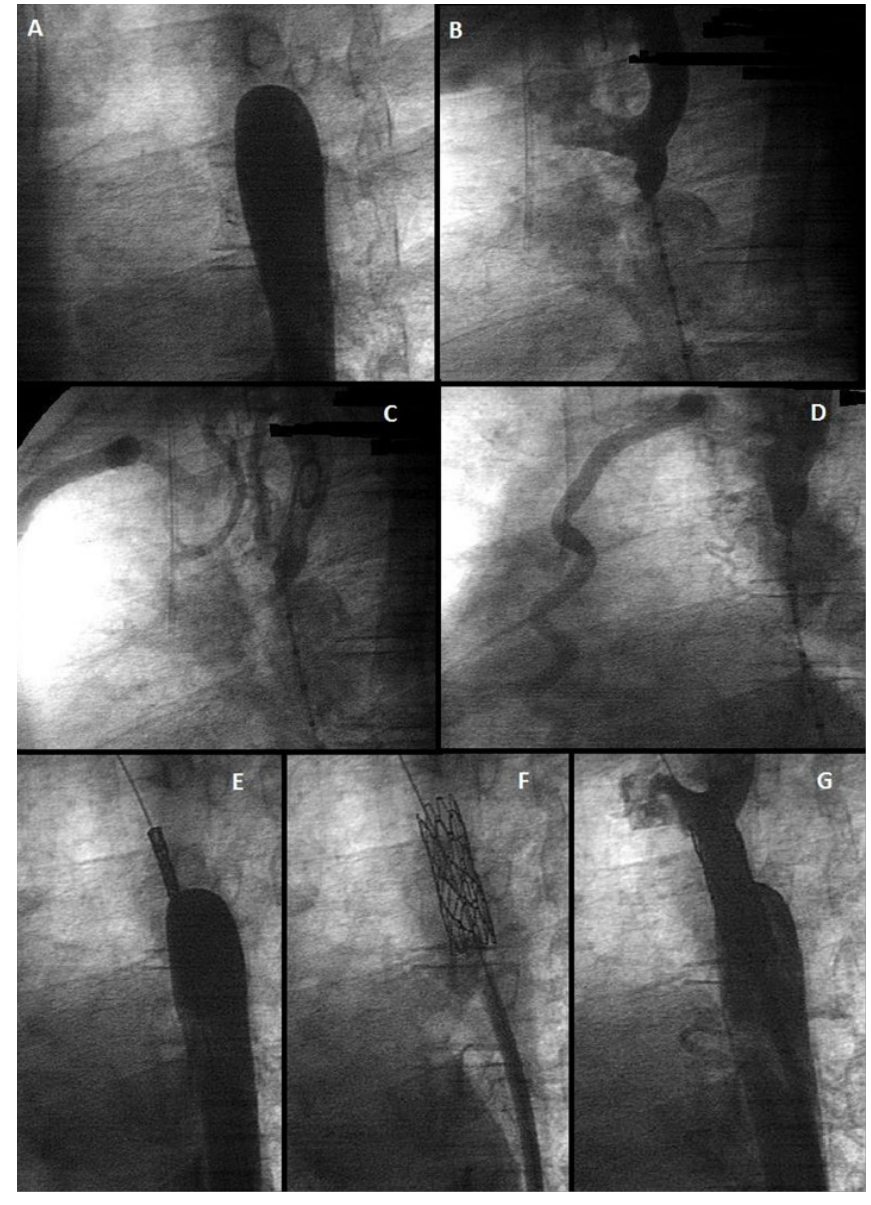

Figure 2: The figure shows the tight aortic coarctation (panels $\mathrm{A}$ and B) with the presence of large collateral circulation arterial bridging the obstacle (panels $C$ and $D$ ). The placement of a covered stent at the level of the coarctation ensured a good recovery of aortic flow (panels E, F and G). For more details see the text.

The image A within figure two shows the aortic coarctation, through retrograde opacisation of descendent aorta, approached from the right femoral artery. The image B shows the aortic arch and the vessels above the aorta distally to the coarted segment. Images C and D illustrate the well-established collateral arterial circulation which allows the blood flow to bypass the obstacle at the coartation level. After careful evaluation, we decided to choose the percutaneous stenting as the best approach to treat this patient condition.

\section{PROCEDURE}

After local anaesthesia, the percutaneous procedure stenting started with an incision in the right inguinal area to surgically isolate the femoral artery. $100 \mathrm{mg} / \mathrm{kg}$ body weight eparin was administered and the aorta was reached retrogradely using a 11 F guidewire from the femoral artery. Angiographic study showed 
a severe native post isthmic aortic coartation with a false lumen (three $\mathrm{mm}$ of diameter, ten $\mathrm{mm}$ pre-stenosis and $23 \mathrm{~mm}$ post stenosis aorta diameter with a pressure gradient max of 80 $\mathrm{mmHg}$ measured at pull back with pigtail five French catheter). The coarted segment was overcome using a Terumo guidewire $(0,035$ inch) which was subsequently positioned into the anonymous artery. A long--sheath Mullins $12 \mathrm{~F}$ was introduced until it reached the thoracic aorta with an exchange guidewire 0,035 inch Amplatz super-stiff. A covered stent [NUMED covered Cheatham-platinum (CP) stent $39 \mathrm{~mm}$ ] pre-fixed on a BIB catheter (Outer balloon 12x40; inner balloon sixx30mm) was introduced. Through sequential inflation- deflation of the inner and outer balloon the covered stent was implanted (see images $E$ and $F$ on figure two). At the end of the procedure, when a proximal and distal optimal sealing was obtained and it was demonstrated no interference with the vessels above the aorta, we sutured the common femoral artery with prolene 6.0 and then the surgical access tissues. A post-procedural angiography showed an excellent flow with a residual gradient of $20 \mathrm{mmHg}$ (image $\mathrm{G}$ of figure two). A $20 \mathrm{mmHg}$ transprotesic gradient, which normally represents the cut-off value above which the CoA stenting or re-stenting is considered, in our case has been considered a good outcome given the complex anatomy and the pre-procedural gradient [2,4]. On discharge patient was given antiplatet therapy (cardioaspirin in association with clopidogrel) and antihypertensive medication (Ca-channel blocker and angiotensin II inhibitors) with a good blood pressure control which was confirmed on a 24 hours Holter done as an outpatient one month later.

Moreover, six month and one year echocardiography and clinical examination follow-up confirmed that no complication had subsided and the residual post-procedure gradient was maintained.

\section{DISCUSSION}

Anthropometric characteristic, age, complete physical growth, the absence of other associated cardiac conditions, lead us to choose the percutaneous procedure as recommended from literature data in similar scenarios [2]. In fact, nowadays, the stenting procedure can be considered a valid treatment for adults with native coarctation or re-coarctation. It is a safe and high effective technique, even if long term follow up data are not yet completely available. Percutaneous stent success rate is usually high (around 90\%) and it is associated with an increased diameter of the narrowed segment, a reduction of the systolic gradient and a better control of the blood pressure
[5]. The most serious complication is the aorta rupture which can be fatal, but it is rare (<two\%), other complications such as the aneurysmatic dilatation, re-stenosis and stent failure can be controlled during and after the procedure [5]. The Haas group has collected the first evidences of percutaneous stents correction of aortic coarctation in infants and adolescents using Cheatham-platinum - CP stent, thought on a small sample [6]. On 6 patients with aortic coarctation (mean age 12.7 years old; five native, one re-coarctation) treated with percutaneous stenting it was seen a reduction in the systolic gradient at the maximum pick from $49 \mathrm{mmHg}$ to three $\mathrm{mmHg}$ $(p<0,001)$ with an increment of $350 \%$ of the coarted site diameter $(p<0.001)$. Furthermore, at the 18 months' follow-up it wasn't seen any complication such as re-coarctation, aortic dissection, aneurysm formation or stent dislocation.

The retrospective, multicentre analysis of 588 procedures performed between 1989 and 2005 by the Congenital Cardiovascular Interventional Study Consortium (CCISC) [7] percutaneous stent correction of aortic coarctation appears an extremely favorable procedure with a success rate of $98.6 \%$ (gradient decreasing to values inferior to $20 \mathrm{mmHg}$ or an increment of the coarctation post-stent/ descendent aorta $>0.8)$.

Complications, which represent $11.7 \%$ and often recur in the same patients, were recorded within 16 years as follows: complications related to the femoral vascular access (2.6\%), aneurysm (2.2\%), aortic dissection (1.5\%), cerebrovascular accidents (one $\%)$, two cases of death $(0.3 \%)$.

Success rate of $100 \%$ have been published from Shah et al. [8]. In 44 patients with aortic coarctation (28 native and 16 re-coarctation; mean age $16 \pm 1.8$ years old) who underwent stenting between May 1995 and February 2005 it had been registered a systolic gradient reduction from $29.2 \pm 1.9 \mathrm{mmHg}$ to $3.7 \pm 0.7 \mathrm{mmHg}$ and an increase of the diameter at the coarctation site from $6.1 \pm 0.5 \mathrm{~mm}$ to $13 \pm 0.5 \mathrm{~mm}$. 13 patients underwent re-dilation or re-stenting procedure, nine patients presented complications. At an average follow-up of $19.8 \pm 3.5$ months, 1 patients developed an aneurysm at the stent site.

Furthermore Tzifa et al. in a work from 2006 evaluated the efficacy of CP covered stent positioning to treat 30 patients affected from complex aortic coarctations (aneurysmatic formation or stent related complications or in at-risk patients for complication associated to the complex anatomy or the age [9]. The procedure was associated to a gradient reduction at the coarctation level and to significant increase of the diameter, at the end of the 40 months' follow-up stents resulted patent 
and in place and in half of the patients the antihypertensive therapy had been reduced or stopped. Similarly, the Bruckheimer group, which studied 22 subjects with native coarctation who underwent stent implantation, registered a significant increase of coarctation diameter (from 3. $6 \pm 1.9$ $\mathrm{mm}$ to $12.6 \pm 1.9 \mathrm{~mm}, \mathrm{p}<0.001$ ) associated to a significant reduction of pick pressure gradient (from $29.4 \pm 8.5$ to 6.7 $\pm 5.7 \mathrm{~mm} \mathrm{Hg}, \mathrm{p}<0.001$ ), with minor complications (small stent rupture associated to further dilations treated with a second stent and a femoral pseudoaneurysm). At the shortterm follow-up (18.5 months) no evidences of re-coarctation or aneurysm were noted [10]. Moreover favorable results emerged from the Chakrabarti et al experience on 102 de-coarctation procedure through percutaneous stent positioning (94 native coarctations, re-coarctation) performed between 2002 and 2008 on 88 patients (mean age 20.6 years old). Published data from this study showed a mean reduction of coarctation gradient from $20 \mathrm{mmHg}$ to $4 \mathrm{mmHg}$; none fatal event, 4 patients developed early complications (1 needed surgical treatment) 2 late complications needed additional dilation stent procedure (average follow up 34.5 months) [11]. Recently the Chang et al group analyzed CP covered stent technique (25 cases) as first line treatment in native aortic coarctations.

Published data showed a significant reduction in the aortic gradient (from $67.5 \mathrm{mmHg}$ to two $\mathrm{mmHg}$ ) and a similar significant increment of the stenotic diameter (from $5.0 \mathrm{~mm}$ to $17.9 \mathrm{~mm}$ ). No complication at the end of the 72 month follow up period was recorded [12].

\section{CONCLUSION}

Percutaneous approach through stent placement to treat native aortic coarctation in adolescents and adults represent a safe and effective procedure. Long term follow-up data will be extremely useful to better define risks and benefits of the procedure, which should be performed in specialist experienced units. [13-16].

Moreover, our case is noteworthy because the young subject in analysis was asymptomatic except for arterial hypertension until the diagnosis was made. This demonstrated the multiple cardiovascular compensation mechanisms, as shown by the extensive collateral circulation developed between the upstream and downstream segment from the stenosis, even in extreme situations such as this.

\section{ACKNOWLEDGMENT}

None

6. Haas NA, Lewin MA, Knirsch $W$ et al. Initial experience using the NuMED Cheatham Platinum (CP) stent for interventional treatment of coarctation of the aorta in children and adolescents. Z Kardiol. 2005;94:113-20.

7. Forbes TJ, Garekar S, Amin Z et al.; Congenital Cardiovascular Interventional Study Consortium (CCISC). Procedural results and acute complications in stenting native and recurrent coarctation of the aorta in patients over 4 years of age: a multi-institutional study. Catheter Cardiovasc Interv. 2007 1;70:276-85.

8. Shah L, Hijazi Z, Sandhu S et al. Use of endovascular stents for the treatment of coarctation of the aorta in children and adults: immediate and midterm results. J Invasive Cardiol. 2005;17:614-8.

9. Tzifa A, Ewert P, Brzezinska-Rajszys $G$ et al. Covered Cheatham-platinum stents for aortic coarctation: early and intermediate-term results. J Am Coll Cardiol. 2006;47:1457-63. 
10. Bruckheimer E, Dagan T, Amir G et al. Covered CheathamPlatinum stents for serial dilation of severe native aortic coarctation. Catheter Cardiovasc Interv. 2009;74:117-23.

11. Chakrabarti S1, Kenny D, Morgan G et al. Balloon expandable stent implantation for native and recurrent coarctation of the aorta--prospective computed tomography assessment of stent integrity, aneurysm formation and stenosis relief. Heart. 2010;96:1212-6.

12. Chang ZP1, Jiang SL, Xu ZY et al. Use of covered CheathamPlatinum stent as the primary modality in the treatment for native coarctation of the aorta. Chin Med J (Engl). 2012;125:1005-9.

13. Ringel RE, Vincent J, Jenkins KJ et al. Acute outcome of stent therapy for coarctation of the aorta: results of the coarctation of the aorta stent trial. Catheter Cardiovasc Interv. 2013;82:503-10.

14. Perera $\mathrm{AH}$, Rudarakanchana N, Hamady M et al. Newgeneration stent grafts for endovascular management of thoracic pseudoaneurysms after aortic coarctationrepair. J Vasc Surg. 2014;60:330-6.

15. Eckroth-Bernard K, Yoon HR, Ryer EJ et al. Percutaneous endovascular repair of adult aortic coarctation. J Vasc Surg. 2014;59:1120.

16. Luijendijk P, Bouma BJ, Groenink M Et al. Surgical versus percutaneous treatment of aortic coarctation: new standards in an era of transcatheter repair. Expert Rev Cardiovasc Ther. 2012;10:1517-31. 